\title{
Collision Avoidance Affected by Walker's Head Direction in a Virtual Environment
}

\author{
Shunya Ueda ${ }^{1}$ and Michiteru Kitazaki ${ }^{2}$ \\ ${ }^{1}$ Graduate School of Engineering, Toyohashi University of Technology, \\ 1-1 Hibarigaoka, Tempakucho, Toyohashi, Aichi, 441-8580, Japan \\ ueda@real.cs.tut.ac.jp \\ ${ }^{2}$ Department of Computer Science and Engineering, \\ Toyohashi University of Technology, \\ 1-1 Hibarigaoka, Tempakucho, Toyohashi, Aichi, 441-8580, Japan \\ mich@cs.tut.ac.jp
}

\begin{abstract}
We investigated participants' active response to avoid collision with an approaching walker in a virtual environment. The walker was approaching and then changed his direction leftward or rightward at a random timing. The walker's head rotated (yaw) leftward or rightward, or remained straight at $533 \mathrm{~ms}$ before direction change of walking. Ten participants were asked to avoid collision by moving a mouse laterally to move own viewpoint. We found that participants' collision avoidance behavior was affected by head directions. They moved in the opposite direction to the other walker's head direction when the walker rotated his head. These results suggest that we utilize other people's head direction to avoid collision in active situation as well as static perceptual situation.
\end{abstract}

Keywords: Perception and action, Collision avoidance.

\section{Introduction}

We can perceive other people's walking direction very quickly and accurately. Its accuracy depends on spatial and temporal information such as presentation duration and body surface [1]. Ten to twenty point lights attached to body joints elicit biological motion perception such as walking and dancing [2]. The accuracy of direction perception of a point-light walker is lower than the gray-shaded computer graphics walker, but high enough [1][3]. Since human walker's gaze and head direction reflect the intended direction of walking [4], human observers utilize other people's eye direction [5] and head direction [3][6] to perceive the other's walking direction. These studies employed cognitive inferences [2] and perceptual judgments [3][6] of static participants, who were observing a walker approaching to the participant in a virtual environment. However, participants' active or behavioral response for approaching walkers has not been investigated. Active responses (actions) and passive perception (judgments) are differently induced by visual stimulation. Actions and manual control decreases visual illusions [7][8] and facilitates some aspects of visual perception [9]. Thus, illusory effects of head direction might be diminished by active responses. 


\section{Methods}

Ten naive observers with uncorrected or corrected normal vision participated in the experiment after written informed consent. The experiment was approved by the Committee for the Human-subject Studies at Toyohashi University of Technology. The stimulus was created and controlled by a computer (DELL, Precision workstation T1600, Intel Core i3-2120 3.30GHz, NVIDIA Quadro FX3700), and projected on a rear-screen (248 wide x 186 high $\mathrm{cm}$ ) by a 3CRT projector (Barco, Cine7/II, 1024 x 768 pixel, $60 \mathrm{~Hz}, 0.0077-9.404 \mathrm{~cd} / \mathrm{m}^{2}$ ). The experiment was performed in a darkroom.

In a virtual environment of a corridor (10m width floor and ceiling with $2.5 \mathrm{~m}$ height left and right walls that were filled with random dots: $10 \mathrm{dots} / \mathrm{m}^{2}$ ), a walker (170 cm tall, Male) approached (2 steps/s, $2.94 \mathrm{~km} / \mathrm{h})$ to participants (Figure 1). The participants also moved ahead at the same speed. The other walker changed his direction randomly leftward or rightward (6.7 deg) at a random timing (1.67-2.67 s) after starting walking. The direction change took $300 \mathrm{~ms}$. The walker's head rotated (yaw) leftward or rightward (20 deg), or remained straight at $533 \mathrm{~ms}$ before direction change of walking. The head turn took $167 \mathrm{~ms}$. These stimuli were presented on the rear-projection screen $(63.60 \times 49.88 \mathrm{deg}$ in visual angle) and participants observed them at $200 \mathrm{~cm}$ viewing distance. All combinations of experimental conditions were repeated 20 times in random orders (Figure 2). Participants were asked to avoid collision by moving a mouse laterally to move their own viewpoint.

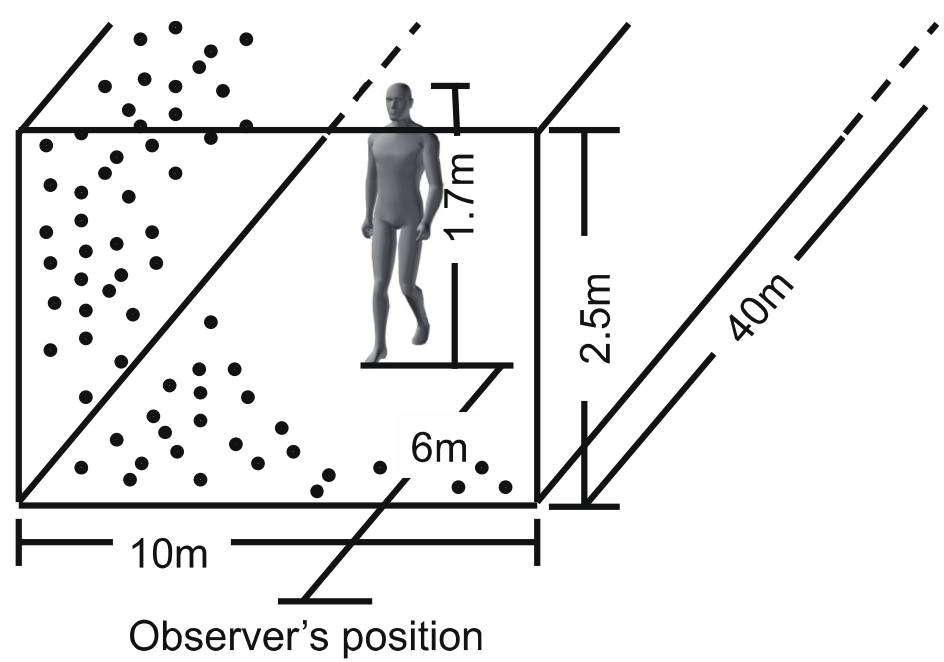

Fig. 1. Schematic of virtual environment. The corridor was made with four walls filled with random dots. Gray-shaded walker approached to the observer/participant. 


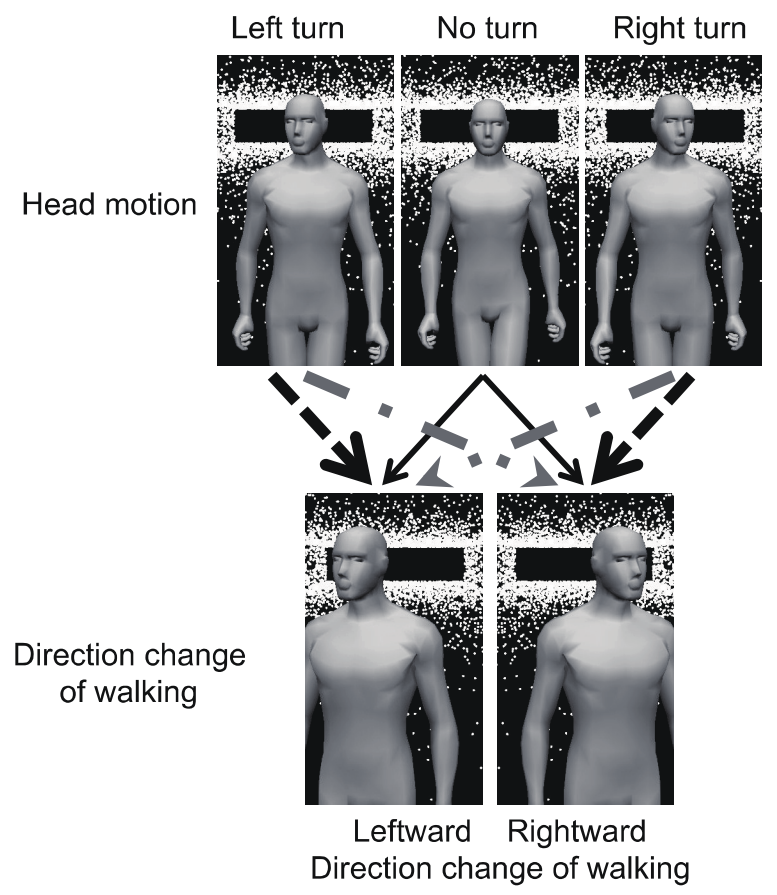

Fig. 2. Experimental conditions. The rotation directions of head and the following direction changes of walker were combined for setting 6 conditions. The combination of the left (right) head turn and the left (right) walking direction change is the consistent condition of head and walking, while the combination of the left (right) head turn and the right (left) walking direction change is the inconsistent condition.

\section{$3 \quad$ Results}

We found that participants' collision avoidance behavior was affected by head directions (Figure 3). They moved in the opposite direction to the other walker's head direction when the walker rotated his head. In the conditions that the head yaw was consistent with the walking direction change, collision avoidance began in the correct direction (opposite direction to the walker) approximately $500 \mathrm{~ms}$ before collision avoidance action in the conditions without head rotation. In the conditions that the head yaw was inconsistent with the walking direction change, collision avoidance began in the wrong direction (same direction as the walker) approximately $500 \mathrm{~ms}$ before the no-head-rotation conditions, then was being returned to the correct direction after $500 \mathrm{~ms}$ of the first action. 


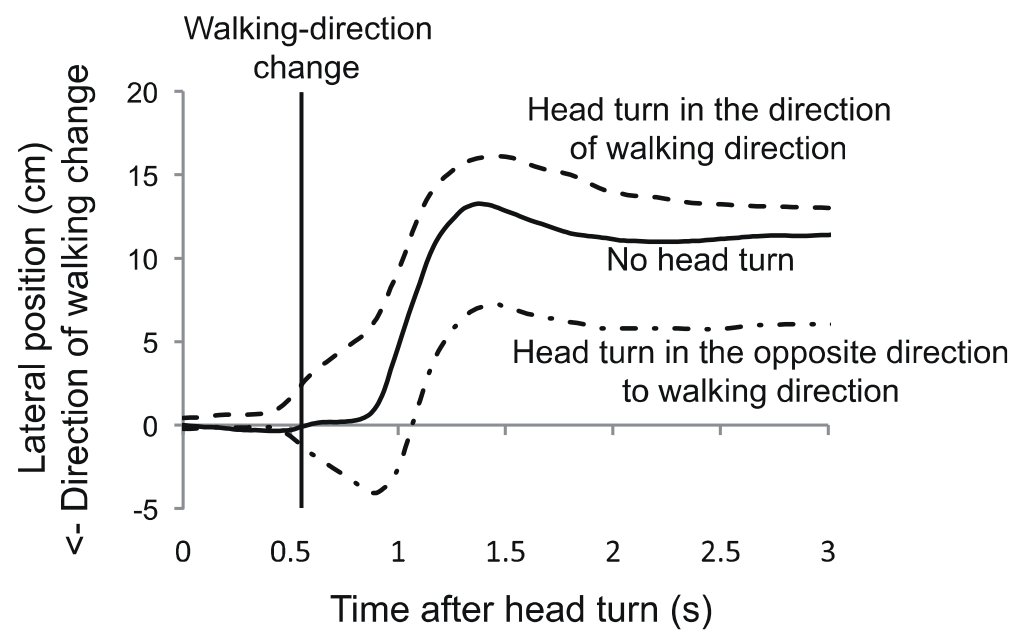

Fig. 3. Experimental results. Lateral position of participant's viewpoint was plotted against time after head turn. Positive value of the vertical axis indicated the opposite direction to walkingdirection change, thus the appropriate direction for collision avoidance.

\section{Discussion}

These results suggest that we utilize other people's head direction to avoid collision in active situation as well as static perceptual situation. Thus, the effect of head rotation on walker's perception is not a visual illusory effect, but rather it supports the notion that our perception of and action to other walkers are related to our social cognition or social perception. Directions of eye and head often reflect walker's intention of walking and path finding. Thus, human observers utilize others' gaze and head direction to perceive and infer others' behavior. This kind of perception and inference seems to be a rudimentary social communication and important for human as social mobile observers. The fact that human perceptual accuracy of other's gaze is very high also supports this notion [10]. Perceptions of other walkers' motion and gaze shift elicit brain activities at the regions relating to social cognition [11][12]. These findings also support the importance of other walker's perception to investigate our social cognition.

Our findings can contribute to design robot' walking motions that facilitate human perception or inference of robot's heading change naturally and automatically. We should put appropriate motions of robot's head and eyes at an appropriate timing before heading change.

Acknowledgments. This research was partly supported by Grant-in-Aid for Scientific Research (B) \#22300076 and Grant-in-Aid for Challenging Exploratory Research \#23650060 from MEXT Japan. 


\section{References}

1. Sato, T., Inoue, Y., Tani, Y., Matsuzaki, N., Kawamura, K., Kitazaki, M.: Perceiving the direction of walking. In: ECVP (European Conference on Visual Perception), Utrecht, Netherlands (2008); Perception 37(suppl.), 27 (2008)

2. Johansson, G.: Visual perception of biological motion and model for its analysis. Perception \& Psychophysics 14(2), 201-211 (1973)

3. Kitazaki, M., Ono, K., Tsukada, Y., Murofushi, Y., Itakura, S.: Perception of walker: Human, robot, and point-lights, Workshop on Human-Robot Symbiosis: Synergistic Creation of Human-Robot Relationships. In: 11th IEEE-RAS International Conference on Humanoid Robots, Bled, Slovenia, October 26 (2011)

4. Hollands, M.A., Patla, A.E., Vickers, J.N.: Look where you're going!: Gaze behaviour associated with maintaining and changing the direction of locomotion. Experimental Brain Research 143, 221-230 (2002)

5. Nummenmaa, L., Hyönä, J., Hietanen, J.K.: I'll walk this way: Eyes reveal the direction of locomotion and make passersby look and go the other way. Psychological Science 20(12), 1454-1458 (2009)

6. Murofushi, Y., Ono, K., Sato, T., Kitazaki, M.: Perceived direction of human, robot and point-light walkers modulated by head direction. In: VSS (Vision Sciences Society Meeting), Naples, Florida, USA, May 13 (2012); Journal of Vision 12(9), 464 (2012), doi:10.1167/12.9.464

7. Goodale, M.A., Milner, A.D., Jakobson, L.S., Carey, D.P.: A neurological dissociation between perceiving objects and grasping them. Nature 349, 154-156 (1991)

8. Harman, K.L., Humphrey, G.K., Goodale, M.A.: Active manual control of object views facilitates visual recognition. Current Biology 9, 1315-1318 (1999)

9. Ichikawa, M., Masakura, Y.: Manual control of the visual stimulus reduces the flash-lag effect. Vision Research 46, 2192-2203 (2006)

10. Gamer, M., Hecht, H.: Are you looking at me? Measuring the cone of gaze. Journal of Experimental Psychology: Human Perception and Performance 33(3), 705 (2007)

11. Pelphrey, K.A., Viola, R.J., McCarthy, G.: When strangers pass: Processing of mutual and averted social gaze in the superior temporal sulcus. Psychological Science 15(9), 598-603 (2004)

12. Morris, J.P., Pelphrey, K.A., Mccarthy, G.: Regional brain activation evoked when approaching a virtual human on a virtual walk. Journal of Cognitive Neuroscience 17(11), 1744-1752 (2005) 